\title{
ACORDO DE NÍVEL DE SERVIÇO PARA MELHORIA DA QUALIDADE NO SUPRIMENTO DE ITENS DE CONSUMO EM UMA INSTITUIÇÃO PÚBLICA DE ENSINO SUPERIOR
}

\section{SERVICE LEVEL AGREEMENT FOR QUALITY IMPROVEMENT IN CONSUMABLES SUPPLY IN A PUBLIC HIGHER EDUCATION INSTITUTION}

\author{
Karoline Santiago de Sousa Silva ${ }^{1}$; Armando Vítor Nascimento Santana ${ }^{2}$; Alberto de Oliveira \\ Cardoso Neto ${ }^{3}$; Denilson Ricardo de Lucena Nunes ${ }^{4}$; André Cristiano Silva Melo ${ }^{5}$ \\ ${ }^{1}$ Universidade do Estado do Pará - UEPA - Belém, Pa/Brasil, \\ karolinesssilva@hotmail.com \\ ${ }^{2}$ Universidade do Estado do Pará - UEPA - Belém/ PA - Brasil \\ armando.santana@grupotpc.com \\ ${ }^{3}$ Universidade do Estado do Pará - UEPA - Belém/ PA - Brasil \\ albertoneeto@gmail.com \\ ${ }^{4}$ Universidade do Estado do Pará - UEPA - Belém/ PA - Brasil \\ denilson.lucena@ibest.com.br \\ ${ }^{5}$ Universidade do Estado do Pará - UEPA - Belém/ PA - Brasil \\ acsmelo@yahoo.com.br
}

\begin{abstract}
Resumo
O objeto de estudo deste artigo foi uma Instituição de Ensino Superior (IES) pública, que apresenta uma gestão de suprimentos baseada na obtenção do melhor resultado em termos de preço e qualidade, conforme definição da lei brasileira. Segundo a legislação brasileira, o principal parametro na seleção de fornecedores para o setor público é o preço, entretanto, os conceitos de qualidade e nível de serviço vão muito além desse aspecto. Os Acordos de Nível de Serviço (ANS), que permitem exigir dos fornecedores outros aspectos que garantam a qualidade no fornecimento de bens ou serviços. A definição desses aspectos depende do tipo de bens ou serviço prrestado. Dessa forma, este artigo teve a finalidade de propôr parâmetros para um ANS afim de melhorar a qualidade no ressuprimento de materiais de consumo de uma IES pública, bem como fornecer elementos para o monitoramento da eficiência de seus fornecedores. Para tanto, foi realizada uma revisão bibliográfica e entrevistas não estruturadas com pessosas importantes no processo de suprimento da instituição estudada. Como resultado foram propostos indicadores de desempenho relacionados com cada problema típico de suprimento encontrado na IES, além disso foram propostas ações de gestão relacionadas as diferentes faixas de desempenho que os fornecedores podem apresentar.
\end{abstract}

Palavras-chave: avaliação de Fornecedores; indicadores de desempenho; Gestão de suprimentos; logística; Indicadores de qualidade.

\section{Introdução}

Apesar dos objetivos e finalidades das empresas públicas e privadas serem distintos, Costa (2000) analisou a gestão de suprimentos nos dois setores e concluiu que os objetivos gerenciais são 
os mesmos, obter o melhor resultado em termos de preço e qualidade, entretanto existem diferenças, e uma delas é a forma como o processo de compras é conduzido. As compras em empresas públicas do Brasil são orientadas pelo art. 37, XXI (BRASIL, 1988) que estabelece a exigência do processo licitatório para a compra ou alienação de bens e a contratração de obras e serviços de qualquer natureza, visando assegurar a igualdade de condições a todos os interessados em vender ou comprar do Estado. Dessa forma, a Lei 8.666, de 21 de junho de 1993, contém a rotina a ser seguida no processo de compra ou contratação por parte das empresas públicas de qualquer esfera no Brasil. O serviço prestado aos órgãos públicos, de interesse em especial para este estudo, é o fornecimento de itens de consumo.

O suprimento de itens de consumo representa uma parcela considerável no orçamento dos governos no Brasil, segundo, os gastos com bens e serviços ficam em torno de $30 \%$, além disso, esses autores, assim como Melo (2012) e Moreno Filho (2012), afirmam que a existe grande esforço na melhoria do processo de compras no setor público, porém outras áreas envolvidas no suprimento ainda carecem maior atenção, como é o caso da Gestão dos Fornecedores. Segundo (Souza, 2011), não é suficiente que os fornecedores no setor público atendam às condições legais para que seu desempenho seja considerado bom. O conceito de eficiência a ser seguido não é apontado em nenhum instrumento legal, portanto, resta seguir o conceito de eficiência como a obtenção do melhor resultado possível com o uso racional dos recursos.

Quanto à gestão de suprimentos, parte das decisões está descritas na Lei 8.666/93, e no que diz respeito à qualidade, o Estado fornece diretrizes através da Instrução Normativa IN/MPOG n.02/2008 para avaliar a qualidade no serviço de terceiros por meio dos ANS (Acordo dos Níveis de Serviço). Dessa forma, tanto para a eficiência quanto para a qualidade no serviço é necessário definir e quantificar métricas, com o intuito de avaliar a eficiência e a qualidade no atendimento, sobretudo nos casos em que não exista um instrumento formal para isso. Recentemente, alguns estudos (Rodrigues et al., 2013; Santos et al., 2013; Silva, 2009) utilizaram essas diretrizes para propor ANS.

Este artigo objetivou propor um modelo de ANS para uma instituição pública (IES), como ferramenta legal para melhoria na qualidade do suprimento, por parte dos fornecedores da instituição, e monitoramento de seus níveis de desempenho neste processo. Assim, o artigo estrutura-se da seguinte forma: Seção 2 que apresenta uma breve revisão bibliográfica sobre compras no setor público, modalidades de compras, acordos de nível de serviço e qualidade no serviço público. Seção 3 onde esta descrita a metodologia utilizada no artigo. A Seção 4 contém uma breve descrição da organização objeto de estudo e como são feitas suas compras. Na Seção 5 são descritos e discutidos os resultados e, por fim, na Seção 6 apresenta-se as conclusões. 


\section{Referencial Teórico}

\subsection{Compras no serviço público}

Segundo Frossard e Câmara (2010), a gestão de suprimentos é um dos principais pilares de qualquer instituição pública, tendo considerável importância estratégica, pois é uma das atividades em que se aplicam os recursos orçamentários existentes, por meio da efetivação dos processos de compras de materiais, bens e serviços necessários ao alcance dos objetivos da instituição. Entendese como gestão de suprimentos: compras, aquisição e guarda dos materiais, bens e serviços necessários à manutenção e ao desenvolvimento das atividades.

A importância das atividades de compras sempre foi presente para as instituições públicas e privadas. Para as instituições públicas ligadas à área de pesquisa científica, essa importância está diretamente interligada ao crescimento das despesas e à complexidade das pesquisas adotadas. A partir da adoção de novas práticas para a gestão institucional, os agentes públicos tendem a reduzir seus custos internos de manutenção (complementar à difusão da informação e do conhecimento), para trazer vantagem competitiva e garantir a realização das estratégias e alcance de objetivos (Batista e Maldonado, 2008).

A Lei de Licitações e Contratos Administrativos, Lei no 8.666, de 21 de junho de 1993 (Brasil, 1993), nos traz o conceito de compras, onde intitula “... como toda aquisição remunerada de bens para fornecimento de uma só vez ou parceladamente".

A área de compras realiza uma atividade primordial no alcance dos objetivos estratégicos da instituição pública, por conta da sua capacidade de afetar a qualidade e entrega de produtos ou serviços essenciais que serão utilizados.

\subsubsection{Modalidades de compras no serviço público}

O processo de compras em uma empresa (pública ou privada) é iniciado a partir das decisões tomadas na gestão de estoques, que têm como objetivo responder a duas questões: Quando comprar? e Quanto comprar? Responder as estas questões, no caso de materiais de consumo, significa analisar um conjunto de variáveis que interferem nos custos relacionados com o volume de estoques, resultante das decisões de quando e quanto comprar.

De acordo com SENA et al. (2010), durante vários anos, o Governo Federal vem trabalhando na evolução e no aperfeiçoamento da legislação, visando desenvolver novas formas de aquisição de bens e serviços, com finalidade de racionalizar os processos, otimizar os contratos, para que os resultados se tornem mais efetivos, sendo as mais usadas: 
- Pregão Eletrônico: Um desses principais processos é o pregão que, de acordo com a Lei $\mathrm{n}^{\circ}$ 10.520/02, que o instituiu, define bens e serviços comuns como, "aqueles cujos padrões de desempenho e qualidade possam ser objetivamente definidos pelo edital, por meio de especificações usuais no mercado". Com isso, se define pregão, Segundo MACHADO, como sendo a modalidade de licitação, que tem por intuito adquirir bens e serviços comuns, por meio de sessão pública, onde são feitos lances e propostas, uma espécie de leilão, não sendo levado em consideração o valor estimado da contratação;

- Sistema de Registro de Preços: de acordo com DIAS et al. (2012), o Sistema de Registro de Preços (SPR) é instrumento que objetiva a realização de contratações sucessivas de bens e serviços, selecionados por meio de licitação, no qual é elaborado um cadastro de produtos e fornecedores para que a Administração Pública contrate de acordo com suas necessidades. A Ata de Registros de Preços (ARP) é um instrumento jurídico vinculativo, obrigatório, com característica de compromisso. Nela se registram as condições estabelecidas para que sejam cobradas posteriormente, em futuras contratações. Qualquer órgão ou entidade pode posteriormente utilizar a ARP.

\subsection{Medidas de qualidade no serviço público}

A visão histórica da qualidade mostra como esta vem sendo conceituada no passar dos anos de forma similar. Por exemplo, a civilização fenícia possuía uma lei a qual afirmava que os soldados fenícios deveriam amputar a mão do fabricante de produtos defeituosos, ou seja, naquela época produtos defeituosos eram aqueles que não estavam conforme as especificações governamentais.

Segundo Slack et al. (2009):

Qualidade é a conformidade, coerente com as expectativas do consumidor; em outras palavras, significa "fazer certo as coisas", mas as coisas que a produção precisa fazer certo variarão de acordo com o tipo de operação.

A qualidade está ligada diretamente aos serviços, processos, sistemas etc. Tudo que gera algum resultado dentro de empresas, organizações e indústrias, leva em consideração a qualidade, visto que esta é um critério de avaliação adotado pela maioria dos consumidores.

A prestação de serviços realizada pelo poder público não mantém o foco na qualidade do serviço, deixando-a relegada a um segundo plano, ainda que as organizações do setor público sejam as responsáveis pela maioria da prestação de bens e serviços à comunidade.

Segundo Martins e Laugeni (2005, p.533), pode-se medir a qualidade dos serviços por meio de três facetas: 
a) Medidas objetivas: muitos elementos da qualidade de um serviço podem ser avaliados somente de maneira subjetiva, mas a empresa precisa estabelecer também medidas objetivas para verificar a qualidade, tais como: Tempo de resposta do serviço, tempo de execução, quantidade de reclamações dos clientes, solicitações por cliente, quantidade de erros;

b) Indicador de qualidade: a empresa também deve procurar estabelecer um indicador de problemas apresentados por seus clientes e monitorá-lo ao longo do tempo;

c) Itens em áreas administrativas. Nestas áreas, os itens mais relevantes para a medida da qualidade são: Disponibilidade (grau de apoio que o fornecedor oferece ao cliente); Atenciosidade (tempo de reação frente à solicitação do cliente); Tempo de atendimento (prazo em que o serviço é realizado); Completibilidade (nível de conclusão do serviço total ou parcial); Tecnicidade (grau de profissionalismo na relação com o cliente).

\subsubsection{Indicadores de desempenho logístico}

Os indicadores de desempenho logístico são medidas que refletem a qualidade do serviço logístico. Esses indicadores permitem identificar possíveis falhas nos processos logísticos, constituindo assim uma ferramenta de análise importante na gestão dos processos logísticos dentro do ambiente organizacional. Segundo Bowersox e Closs (2010), a relação entre excelência logística e medição de desempenho é forte e o monitoramento do desempenho logístico deve estar orientado para processos e não para perspectivas funcionais. O principal objetivo da implementação de sistemas de medição de desempenho (SMD) é monitorar, controlar e direcionar as operações logísticas. A busca por eficiência tem como pré-requisito a alta qualidade dos serviços prestados ao cliente final (Fleury, Wanke e Figueiredo, 2000). No entanto, atualmente para se atingir esse objetivo não basta apenas ter o aprimoramento das atividades internas da empresa. É fundamental também que exista um alto nível de integração entre os parceiros de uma mesma cadeia.

Para BARBOSA, MUSETTI e KURUMOTO (2006), os indicadores de desempenho mais usados nos serviços logísticos se referem ao ciclo de pedido, disponibilidade de estoques, restrições ao tamanho do pedido, facilidade para a colocação do pedido, frequência de entregas, confiabilidade da entrega, qualidade da documentação, procedimentos para reclamações, atendimento completo do pedido, suporte técnico e informação sobre a posição do pedido. Ainda de acordo com esses autores, o desempenho logístico é multidimensional, envolvendo vários objetivos de desempenho, e por esse motivo, nenhum indicador é suficiente para medir esse desempenho. Entretanto, o objetivo dos pesquisadores e gerentes é encontrar um conjunto de indicadores que, coletivamente, capturem a maior parte possível, se não toda, das mais importantes dimensões do desempenho a longo e curto prazo. 
Barbosa, Musetti e Kurumoto (2006) afirmam que a logística deve ser considerada uma atividade de gestão, sendo importante que se desenvolva sistemas de medições próprios, de acordo com suas características e peculiaridades. A definição desses indicadores de desempenho é considerada de diferentes formas pelos pesquisadores. Nesse caso, a estrutura do indicador deveria ter: a) Elemento, que corresponde ao assunto base para a caracterização do indicador; b) Fator, que corresponde à combinação de elementos e c) Métricas, que se refere à unidade de medida utilizada no fator ou elemento.

A abordagem de Christopher (2010) está estruturada em benchmarking e custos. O autor explicita que é necessário avaliar esses custos, provenientes das mudanças das decisões logísticas. Dentre esses indicadores que podem ser usados, o autor cita: os custos totais de distribuição; os custos de transporte; os custos de armazenagem; os custos de processamento de pedido e os custos de entregas locais. Com relação à estruturação do indicador de benchmarking, é preciso avaliar o ambiente competitivo no qual a empresa se encontra, com isso, o autor define três dimensões: os clientes (a base da avaliação), o desempenho de outras empresas e, por último, a comparação desses desempenhos.

As empresas cada vez mais estão conscientizando-se de que não é possível atender às exigências de serviço dos clientes e, simultaneamente, cumprir com os objetivos de custos da empresa, sem trabalhar de forma coordenada com outros participantes da cadeia de suprimentos (Fleury, Wanke e Figueiredo, 2000). Assim, os indicadores de desempenho logístico podem monitorar a qualidade das atividades logísticas internas à empresa ou de seus parceiros (fornecedores). Esses sistemas de medição de desempenho, segundo Bowersox e Closs (2010), são divididos em medidas ou indicadores de desempenho externos e internos.

Os indicadores de desempenho externos são voltados para o cliente, ou seja, qual a percepção do cliente, e para obter conhecimentos inovadores das empresas concorrentes, o benchmarking. As medições típicas da primeira medida, ou indicadores, incluem: desempenho do tempo de ciclo, disponibilidade de informações, suporte aos produtos e resolução de problemas. Quanto ao benchmarking, pode-se dizer que este representa um aspecto crítico para compreender a medição de desempenho e a maioria das organizações utiliza-se dessa técnica.

Os indicadores internos de desempenho estão voltados à comparação de processos e atividades baseados em uma série histórica e são classificados em cinco categorias: a) Custos, que seria o custo real incorrido para atingir objetivos operacionais específicos; b) Serviço ao cliente, examinando a capacidade organizacional de satisfazer aos seus clientes; c) Produtividade, que é a relação entre o resultado produzido (outputs) e a quantia de insumos usados pelo sistema para gerar tal resultado; d) Mensuração de ativos, que constitui a 
contabilização total dos ativos; e e) Qualidade, bem orientado aos processos e projetado para determinar a eficácia de um conjunto de atividades, ao invés de atividades individuais isoladamente.

Evidentemente, dentro de uma empresa, existem vários processos logísticos. No entanto, o acompanhamento de indicadores para todos não é recomendado, sob pena de tornar o processo de coleta de dados demasiadamente complexo e dificultar a tomada de decisões diante de informações dispersas. Nesta pesquisa, os indicadores de desempenho logístico internos compreendem 4 áreas chaves: Atendimento do Pedido ao Cliente; Gestão de Estoques; Armazenagem; Gestão de Transportes.

\subsection{Acordo de nível de serviço}

Acordos dos Níveis de Serviços (ANS) definem serviços providos, produtos que são apoiados, níveis de desempenho, medidas, critérios de relatórios e padrões de qualidade. Na maioria dos casos, ANS são tipicamente complementados com outros documentos de contratos que, juntos, cobrem múltiplos itens como ações corretivas, penalidades e cláusulas de incentivo, falta de desempenho, quantificação dos serviços aceitáveis, critérios de término, propriedade intelectual, procedimentos de resolução de disputa. Numa visão global, um ANS define um conjunto completo de responsabilidades e obrigações entre um provedor de serviços e seu cliente (ASPIC apud FIGUEIREDO, 2002).

Segundo Magalhães e Pinheiro (2007), os benefícios decorrentes da implementação de ANS são: Visão do nível de desempenho dos serviços por ambas as partes envolvidas; Monitoração e revisão dos processos e do desempenho dos serviços; Eliminação dos mal-entendidos entre clientes e fornecedores; Alinhamento das expectativas dos clientes e usuários com os fornecedores; e Estabelecimento de um processo de melhoria contínua.

O ANS dispõe de dois elementos de apoio e controle para que seja cumprindo, sendo eles: Acordo de nível operacional (ANO), que é um acordo entre um provedor de serviço e outra parte da mesma organização, para suportar a entrega do serviço de ao cliente. O ANO define os produtos ou os serviços a serem fornecidos e as responsabilidades de ambas as partes (MAGALHÃES; PINHEIRO, 2007). De maneira a garantir que os ANS firmados entre a área provedora do serviço e as áreas clientes da organização sejam cumpridos, o outro elemento é o Contrato de Apoio (CA), que é um contrato firmado entre o provedor de serviços de e um terceiro. $O$ terceiro fornece bens ou serviços que suportam a entrega do serviço ao cliente. O contrato de apoio define metas e responsabilidades que são necessários para cumprir as metas de nível de serviço pactuadas no ANS (MAGALHÃES; PINHEIRO, 2007). 
Em contratações públicas, o nível de serviço contratado é definido em duas seções do termo contratual, as quais são: modelo de prestação de serviços e modelo de gestão do contrato. O modelo de prestação de serviços consiste na definição dos resultados requeridos do fornecedor e das atividades necessárias para realizá-los, sua forma de realização e mensuração e sua compatibilidade com o plano de cargos da organização pública em questão (CRUZ, 2008). Nesse sentido, é possível afirmar que este abarca a definição de processos a serem executados, de produtos a serem entregues, as metas de serviço a serem alcançadas, entre outros elementos que especificam como o serviço deve ser prestado pelo fornecedor.

O modelo de gestão do contrato consiste na definição de todos os procedimentos, papéis e responsabilidades que terão lugar durante a execução contratual, as formas de mensuração e pagamento dos serviços, o tratamento da segurança da informação, da propriedade intelectual, as responsabilidades comerciais, fiscais, trabalhistas e previdenciárias, as cláusulas de apelação e as condições de encerramento do contrato (CRUZ, 2008).

\section{Método de pesquisa}

Uma pesquisa pode ser classificada quanto a sua natureza, abordagem, objetivo e procedimentos técnicos. A classificação, apresentada em Silva e Menezes (2005), foi usada para tipificar a pesquisa realizada, de acordo com cada critério. Assim, quanto à sua natureza, esta pesquisa foi classificada como Aplicada, pois foi voltada para uma aplicação prática dos conceitos de rede de suprimentos e indicadores de desempenho logístico, associados à solução de um problema específico observado na prática.

Quanto à abordagem, a pesquisa foi classificada como Qualitativa, pois fez uso de entrevistas não estruturadas para conhecer os elementos componentes do processo estudado. Do ponto de vista dos seus objetivos, a pesquisa foi classificada tanto como Exploratória como Descritiva, pois visou tornar explícito o problema, através do levantamento de dados bibliográficos e criação de hipóteses para a análise. Do mesmo modo que tentou descrever certas características importantes do fenômeno e estabelecer relações entre as diversas variáveis associadas.

Finalmente, com relação aos procedimentos técnicos, a pesquisa foi classificada como Estudo de Caso, pois envolveu um estudo exaustivo de poucos objetos de análise, permitindo seu amplo e detalhado conhecimento. Para realização deste estudo foram seguidos alguns passos de pesquisa relacionados à elaboração de uma metodologia de gestão de suprimentos, resultado final de um processo de coleta de dados, a saber: 
a) Elaborar o mapeamento das atividades envolvidas no processo de reposição de materiais de consumo da empresa: entrevistas não estruturadas com os agentes envolvidos no processo de gestão de estoques e compras permitiram elaborar um desenho do processo de suprimentos;

b) Fazer um levantamento do sistema de informação disponível na empresa, verificando a disponibilidade de dados relevantes no processo de gestão de estoques: entrevistas não estruturadas e inspeção local permitiram verificar a existência de banco de dados, bem como a natureza e a relevância dos dados disponíveis para o objetivo geral da pesquisa;

c) Avaliar o planejamento da reposição de materiais atualmente desenvolvido na empresa: Através de entrevista não estruturada ou inspeção local foi verificada a política de resuprimento aplicada na empresa, para criação de um cenário de comparação com as propostas de melhorias;

d) Propor indicadores de desempenho logístico como ferramenta de análise e controle do suprimento nas unidades da IES estudada;

e) Propor questionário estruturado para ser aplicado juntamente aos responsáveis dos pedidos de cada campi da IES, com o intuito de, após a análise dos dados coletados, verificar quantitativamente, por meio de análises estatísticas, a situação do suprimento nas unidades.

\section{Indicadores de desempenho logístico propostos}

A empresa pública, objeto desta proposta, é uma Instituição de Ensino Superior (IES) que possui uma estrutura composta por 20 campi que atuam em 50 municípios do estado do Pará. Essa IES demanda grande quantidade de material de consumo, que após o processo de licitação é entregue no Almoxarifado, órgão responsável por distribuir esses itens para as unidades da capital e do interior.

Um dois objetivos desta pesquisa foi buscar quais problemas são mais comuns, no que diz respeito à qualidade no suprimento dessas unidades e, para isso, foram feitas entrevistas não estruturadas com o responsável do almoxarifado, afim de observar os principais problemas existentes no suprimento da IES. A seguir, foi elaborado um questionário estruturado, conforme o Quadro 1, que serviu de base para a proposição dos indicadores de desempenhos adotados no ANS.

Este questionário teve o objetivo de servir de base para a proposição dos indicadores de desempenho logístico, a serem utilizados como base para o monitoramento e controle da qualidade do ressuprimento no Almoxarifado da instituição. O questionário também abordou uma questão que reflete o nível de importância dos indicadores entre si, com a finalidade de servir de base aos limites toleráveis para cada indicador proposto. 
Após o preenchimento do questionário, por parte do responsável pelo Almoxarifado, foi possível propor indicadores de desempenho no suprimento, relacionado aos problemas identificados a partir da entrevista não estruturada, ambos estão explicitados no Quadro 2.

Quadro 1 - Questionário estruturado para o responsável pelo Almoxarifado Marque as alternativas conforme sua experiência na instituição de forma que: a - Sempre acontece. b - Acontece mais de 50\% dos casos. c-Acontece menos de 50\% dos casos. d-Nunca acontece.

1 ) Com que frequência os itens solicitados chegam com a especificação correta, na quantidade solicitada e no momento acertado?

2 ) Existem itens não atendidos por pedido?

3 ) Existem desconformidades de quantidade nos itens entregues?

4 ) Existem pedidos que chegam atrasados?

5 ) Existem desconformidades de especificações?

6) Os materiais fornecidos são disponibilizados de forma organizada, no ato da entrega?

7) O funcionário do fornecedor se comunica com clareza e facilita o processo de conferência e recebimento?

8) O fornecedor responde rápido através do canal de comunicação existente?

6) Dê sua opinião sobre o grau de importância relativa entre os quesitos a seguir. A importância será expressa por uma escala de 1 (menos importante) até 4 (mais importante), de forma que, se um ou mais quesitos possuírem a mesma importância você deve representar isso repetindo suas notas.

Itens atendidos por pedido

Itens entregues em conformidade de quantidade

Itens entregues em conformidade de especificações

Pedidos entregues dentro do prazo acertado

Fonte - Autores (2015)

Quadro 2 - Problemas no suprimento e indicadores de desempenho propostos

\begin{tabular}{|l|l|}
\hline \multicolumn{1}{|c|}{ Problemas Observados } & \multicolumn{1}{|c|}{ Indicadores Propostos } \\
\hline $\begin{array}{l}\text { Pedidos que são entregues com desconformidade, ou seja, } \\
\text { em quantidades superiores ou inferiores as que foram } \\
\text { solicitadas; }\end{array}$ & $\begin{array}{l}\text { Porcentagem de itens em conformidade } \\
\text { de quantidade por pedido }\end{array}$ \\
\hline $\begin{array}{l}\text { Pedidos entregues com itens fora das especificações } \\
\text { solicitadas }\end{array}$ & $\begin{array}{l}\text { Porcentagem de itens em conformidade } \\
\text { de especificação por pedido }\end{array}$ \\
\hline
\end{tabular}




\begin{tabular}{|l|l|}
\hline \multirow{2}{*}{$\begin{array}{l}\text { Pedidos não atendidos, ou seja, não houve reposição } \\
\text { daqueles itens pedidos. }\end{array}$} & $\begin{array}{l}\text { Porcentagem de pedidos entregues no } \\
\text { prazo }\end{array}$ \\
\cline { 2 - 2 } & $\begin{array}{l}\text { Porcentagem de itens atendidos por } \\
\text { pedido }\end{array}$ \\
\hline
\end{tabular}

Fonte - Autores (2015)

\subsection{Proposta para o ANS}

O ANS, por se tratar de um contrato, deve conter em seu texto, de forma explícita, a descrição dos indicadores, bem como a maneira de obtê-los. Assim, os indicadores propostos no Quadro 2 poderão ser calculados a partir do registro de informações obtidas no decorrer do processo de fornecimento dos itens de consumo. O Quadro 3 contêm as formulações para o cálculo dos indicadores propostos.

\section{2. Índice de desempenho e medidas de ação}

O índice de desempenho proposto foi obtido a partir dos indicadores descritos no Quadro 3. A compilação desses indicadores em um único número se dá por uma média ponderada, onde os pesos de ponderação refletem o grau de importância desses indicadores, conforme a percepção do gestor do Almoxarifado.

Quadro 3 - indicadores de desempenho propostos

\begin{tabular}{|c|c|c|c|}
\hline Indicador & Freq. & Fórmula & Detalhamento \\
\hline $\begin{array}{l}\text { Porcentagem de } \\
\text { itens em } \\
\text { conformidade de } \\
\text { quantidade por } \\
\text { pedido }\end{array}$ & $\begin{array}{c}\text { A cada } \\
\text { recebimento }\end{array}$ & $\ln d_{1}=$ & $\begin{array}{l}Q_{\text {rec }}=\text { Quantidade total de itens } \\
\text { recebidos por pedido; } Q_{\text {conf }}= \\
\text { Quantidade total de itens recebidos } \\
\text { em conformidade por pedido. }\end{array}$ \\
\hline $\begin{array}{l}\text { Porcentagem de } \\
\text { pedidos entregues no } \\
\text { prazo estipulado }\end{array}$ & Semestral & $\overline{\operatorname{Ind}}_{2}=\left(Q_{p r z} / Q_{r e c}\right)$ & $\begin{array}{l}Q_{\text {prz }}=\text { Quantidade total pedidos } \\
\text { entregues no prazo; } Q_{r \in c}= \\
\text { Quantidade total de pedidos } \\
\text { recebidos. }\end{array}$ \\
\hline $\begin{array}{l}\text { Porcentagem de } \\
\text { itens atendidos por } \\
\text { pedido }\end{array}$ & $\begin{array}{c}\text { A cada } \\
\text { recebimento }\end{array}$ & $\operatorname{lnd}_{3}=\left(Q_{\text {atnd }} / Q_{\text {sol }}\right)$ & $\begin{array}{l}Q_{\text {sol }}=\text { Quantidade total de itens } \\
\text { solicitados no pedido; } Q_{\text {atnd }}= \\
\text { Quantidade total de itens atendidos } \\
\text { no pedido. }\end{array}$ \\
\hline
\end{tabular}




\begin{tabular}{|c|c|c|c|}
\hline $\begin{array}{l}\text { Porcentagem de } \\
\text { itens em } \\
\text { conformidade de } \\
\text { especificação por } \\
\text { eedido }\end{array}$ & $\begin{array}{c}\text { A cada } \\
\text { recebimento }\end{array}$ & $\operatorname{Ind}_{4}=\left(Q_{\text {espo }} / Q_{\text {itmrec }}\right)$ & $\begin{array}{l}Q_{\text {espo }}=\text { Quantidade total de itens em } \\
\text { conformidade de especificação no } \\
\text { pedido; } Q_{\text {itmrec }}=\text { Quantidade total } \\
\text { de itens recebidos no pedido. }\end{array}$ \\
\hline
\end{tabular}

Fonte - Autores (2015)

No presente estudo de caso, esses pesos foram retirados do questionário do Quadro 1. Com a atribuição do grau de importância dos indicadores entre si, a partir da análise do responsável pelo almoxarifado, a fórmula segue a Equação 1.

$$
I D=\frac{100}{\sum p_{i}} \sum p_{i} \overline{\operatorname{lnd}}_{i}
$$

sendo $I D$ o índice de desempenho do fornecedor nos últimos seis meses, $\overline{\operatorname{Ind}}_{i}$ o valor médio do indicador, obtido no último período de seis meses, e $p_{i}$ o peso de ponderação correspondente do indicador. Deve-se ressaltar que o indicador $\overline{\operatorname{Ind}}_{2}$ é o único que já representa a média para o período, os demais indicadores encontrados no Quadro 3 são processados a cada entrega, portanto a média para os últimos seis meses deve ser calculada considerando o número de recebimentos neste período. Os pesos de importância adotados pelo responsável do Almoxarifado da IES foram: 2 para Itens atendidos por pedido, 3 para Itens entregues em conformidade de quantidade, 4 para Itens entregues em conformidade de especificações e 4 para Pedidos entregues dentro do prazo acertado.

A partir do valor calculado de $I D$, considerando os pesos adotados na IES estudada e os possíveis valores dos indicadores, propõe-se medidas de ação para cada faixa de valor encontrada: entre 0\% - 49\% - Descredenciamento por não cumprimento do nível de desempenho estabelecido no contrato, entre 50\% - 59\% - Multa, conforme estabelecido no contrato, entre $60 \%$ - 79\% Notificação do fornecedor e exigência de um plano para correção das falhas. O plano deverá ser aprovado e acompanhado pela contratante, entre $80 \%$ - 94\% - Notificação e observação e entre $95 \%$ - $100 \%$ - Apto para o fornecimento.

\section{Conclusões}

A contratação por ANS está sendo vista pelas empresas como uma manobra viável para solucionar os problemas decorrentes da terceirização. A gestão dos ANS embasada no acompanhamento de indicadores de desempenho é apontada pela literatura como uma metodologia eficiente de estabilização do serviço prestado pelos fornecedores. Por conta da escassa literatura sobre a aplicação desta técnica em empresas públicas, colocamo-nos na linha de pesquisadores que compartilham os benefícios da importação desses modelos do setor privado, salvo a demanda de 
adaptações que são necessárias. Este trabalho abordou exatamente esta prática, na forma de estudo de caso, contribuindo para a literatura do setor público específica, levantando indícios de que a utilização de ANS é possível no serviço público e pode resultar em ganhos para instituições públicas, no caso a IES pública considerada.

Abstract: The study object this product was a public Higher Education Institution (HEI) that has a Supply Management based on achieving the best results in terms of price and quality, as the definition of Brazilian law. According to Brazilian legislation, the principal parameter on the Source Selection for public sector is the price, however, the quality concepts and service level goes further from that aspect. Service Level Agreements (SLA), which allow require other suppliers aspects that guarantee quality of goods services or supply. The definition of these aspects depends on the type of goods or services are proved. Thus, this article aimed to propose parameters for a SLA in order to improve quality in resupply of consumables of a public HEI as well as provide input for monitoring the efficiency of their suppliers. To this end, a literature review and interviews with important people in the procurement process of the studied institution was performed. As a result, have been proposed performance indicators related to each typical supply problem encountered in HEI also been proposed management actions concerning the different performance ranges that suppliers may present.

Keywords: Supplier evaluation; performance indicators; Supply management; logistics; Quality indicators.

\section{Referências}

BARBOSA, D. H.; MUSETTI, M. A.; KURUMOTO, J. S. Sistema de medição de desempenho e a definição de indicadores d e desempenho para a área de logística. XIII SIMPEP - Simpósio de Engenharia de Produção, 2006, Bauru/SP. Anais... Disponível em $<$ http://www.simpep.feb.unesp.br/anais/anais_13/artigos/779.pdf $>$. Acesso em: 17 de dezembro de 2014.

BATISTA, M. A. C.; MALDONADO, J. M. S. V. O papel do comprador no processo de compras em instituições públicas de ciência e tecnologia em saúde (C\&T/S). Revista de Administração, v.42, n.4, p.681-699, Rio de Janeiro, julho/agosto 2008 .

BOWERSOX, D.J. \& CLOSS, D.J. Logística empresarial: o processo de integração da cadeia de suprimento. São Paulo: Atlas, 2010.

BRASIL. Lei n. 8.666, de 21 de junho de 1993. Regulamenta o art. 37, inciso XXI, da Constituição Federal, institui normas para licitações e contratos da Administração Pública e dá outras providências. Diário Oficial, Brasília, Distrito Federal, 22 jun. 1993. Disponível em: 〈www.planalto.gov.br〉. Acesso em: fev. 2015.

CHRISTOPHER, Martin. Logística e gerenciamento da cadeia de suprimentos: criando redes que agregam valor. 2 ed. São Paulo: Cengage Learning, 2010.

COSTA, L. C. Sistemas de compras privadas e públicas no Brasil. Revista de Administração, v.35, n.4, p.119-128, São Paulo, out/dez 2000.

CRUZ, C. S. Governança de TI e conformidade legal no setor público: um quadro referencial normativo para a contratação de serviços de TI. 2008. 252f. Dissertação (Mestrado em Gestão do Conhecimento e da Tecnologia da Informação). Universidade Católica de Brasília, Brasília, 2008. Disponível em: . Acesso em: 15 dez. 2009.

DIAS, H. A. S. Sistema de registro de preços: aspectos controvertidos da figura do "carona”. Revista TCMEG. 2012.

FLEURY, Paulo F. WANKE, Peter. FIGUEIREDO, Kleber F. (Org). Logística Empresarial: a perspectiva brasileira. São Paulo. Ed. Atlas. 2000. 
Frossard, L. B. de M; Leonor Moreira CÂMARA, L. M. Poder de Compras da Administração Pública Federal: Novas Perspectivas para o Debate Acadêmico. Encontro de Administração Pública e Governança - EnAPG. Associação Nacional de Pós-graduação e Pesquisa em Administração - ANPAD. VITÓRIA, ES: 2010. Disponível em: <www.anpad.org.br/diversos/trabalhos/EnAPG/enapg_2010/2010_ENAPG393.pdf >. Acesso em: fev. 2015.

MACHADO, J. P. P. O pregão como modalidade de licitação. Escola de Direito e Relações Internacionais, Complexo de Ensino Superior do Brasil - UniBrasil.

MAgAlHÃES, I. L.; PINHEIRO, W. Gerenciamento de Serviços de TI na Prática: uma abordagem com base na ITIL. 1.ed. São Paulo: Novatec, 2007.

SENA, C. B. et al. Licitação Por Pregão Eletrônico: economicidade, celeridade e transparência na Secretaria de Estado da Fazenda do Pará - SEFA. VII Simpósio de Excelência em Gestão e Tecnologia. 2010.

SlACK, N, StUART, C, JOHnStON, R. Administração da Produção. $3^{\circ}$ Edição, São Paulo, Atlas, 2009.

\section{Dados dos autores:}

Nome completo: Karoline Santiago de Sousa Silva

Filiação Institucional: Universidade do Estado do Pará, UEPA

Departamento: Engenheira de Produção,

Endereço completo para correspondência: Travessa Enéas Pinheiro, 2626. Belém - Pa. Brasil.

Telefones para contato: (55) 91 3276-4011/3131-1907

e-mail: karolinesssilva@hotmail.com

Nome completo: Armando Vítor Nascimento Santana

Filiação Institucional: Universidade do Estado do Pará, UEPA

Departamento: Engenheira de Produção,

Endereço completo para correspondência: Travessa Enéas Pinheiro, 2626. Belém - PA. Brasil.

Telefones para contato: (55) 91 3276-4011/3131-1907

e-mail: armando.santana@grupotpc.com

Nome completo: Alberto de Oliveira Cardoso Neto

Filiação Institucional: Universidade do Estado do Pará, UEPA

Departamento: Engenheira de Produção,

Endereço completo para correspondência: Travessa Enéas Pinheiro, 2626. Belém - PA. Brasil.

Telefones para contato: (55) 91 3276-4011/3131-1907

e-mail: albertoneeto@gmail.com

Nome completo: André Cristiano Silva Melo

Filiação Institucional: Universidade do Estado do Pará, UEPA 
Função ou cargo ocupado: Professor D.Sc. em Engenharia de Produção, e Coordenador dos laboratórios CONCEPT e NILO

Endereço completo para correspondência: Travessa Enéas Pinheiro, 2626. Belém - PA. Brasil. Telefones para contato: (55) 91 3276-4011/3131-1907

e-mail: acsmelo@yahoo.com.br

Nome completo: Denilson Ricardo de Lucena Nunes

Filiação Institucional: Universidade do Estado do Pará, UEPA

Função ou cargo ocupado: Professor D.Sc. em Engenharia de Produção.

Endereço completo para correspondência: Travessa Enéas Pinheiro, 2626. Belém - PA. Brasil. Telefones para contato: (55) 91 3276-4011/3131-1907

e-mail: denilson.lucena@ibest.com.br

Submissão: $11 / 09 / 2015$

Aceito: $14 / 09 / 2015$ 